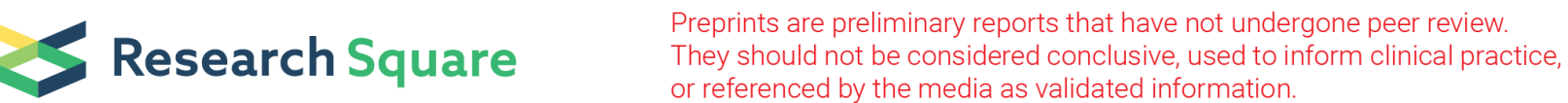

\section{Cervical Cutaneous Tuberculosis Shows the Inflammatory Infection Environment of Local Necrotic Granulation Tissue through the Expression Profile of Chemokine}

\section{Zihui Huang ( $\nabla$ candy73zihui@sina.com )}

Nanjing Integrated Hospital of Traditional Chinese and Western medicine Affiliated to Nanjing University of Traditional Chinese Medicine

Jiayan Qian

Nanjing Integrated Hospital of Traditional Chinese and Western medicine Affiliated to Nanjing University of Traditional Chinese Medicine

Lujue Gao

Lianqing Hong

Yazhou Li

Yang Yu

Sixun Zhu

Minxing Gao

Research article

Keywords: Cervical Cutaneous Tuberculosis; IncRNA microarray; Fluorescence real-time quantitative PCR;CXCR3;CXCL9;CXCL10;CXCL11

Posted Date: November 6th, 2019

DOI: https://doi.org/10.21203/rs.2.16910/v1

License: (c) (1) This work is licensed under a Creative Commons Attribution 4.0 International License. Read Full License 


\section{Abstract}

Background Tuberculosis is an important infectious disease that jeopardizes human health. Research on extrapulmonary tuberculosis is still rare, especially cutaneous tuberculosis, a more refractory extrapulmonary tuberculosis. This study is to explore the inflammatory and immunobiomarkers of patients with cutaneous tuberculosis and to analyze the potential functions and pathways involved in the pathogenesis of $\mathrm{M}$. tuberculosis (Mtb) infection, in order to support cutaneous tuberculosis diagnosis and treatment.Methods Analysis of mRNA identifies biomarkers in cutaneous tuberculosis and nonspecific infectious ulcer tissue. Analyze the correlation between gene ontology (GO) and the Kyoto Gene and Genomic Encyclopedia (KEGG) biological pathways, immunity, inflammation and chemokines. Chemotaxis factors such as CXCL9, CXCL10, CXCL11 and CXCR3 were compared by quantitative PCR analysis.Results The mRNA expression levels of CXCL9, CXCL10, CXCL11 and CXCR3 in local necrotic granulation tissue of cutaneous tuberculosis were significantly increased compared with those of normal tissues. GO analysis indicated that activation of various immune responses and inflammatory responses may be associated with up-regulation of chemokine genes. Gene Ontology (GO) and Kyoto Biogenomics and Genomics Encyclopedia (KEGG) biological pathway analysis revealed the relationship between immune, inflammatory and chemokine pathways.Conclusion GO analysis and KEGG analysis of differentially expressed mRNA revealed potential functions and pathways associated with the onset of Mtb infection.

\section{Background}

Cutaneous tuberculosis are caused by M. tuberculosis invading collective local tissues through various routes of transmission, and eventually lead to necrosis of the surrounding soft tissue, subcutaneous and pericutaneous, and cause chronic wounds formed by liquefaction and ulceration. It is the most common type of specific infectious ulcer in the clinic,which belongs to extrapulmonary tuberculosis and is one of the world's leading infectious diseases and an important part of tuberculosis (TB).

According to the routes of transmission, cutaneous tuberculosis can be divided into the following three types: endogenous tubercu[1]losis transmission, exogenous M. tuberculosis infection and bacillus Calmette-Guérin(BCG)strong reaction. Among them, the clinical spread of endogenous tuberculosis is the most common, that is, tuberculosis such as lymphatic tuberculosis, bone and joint tuberculosis, breast tuberculosis, etc. directly spread to adjacent skin tissues, or through ulcerated skin through blood, and lymphatic tissue is damaged. Cutaneous tuberculosis occur in the neck, underarms and perianal. Because the skin surface is weak, the nerves and blood vessels are densely distributed, and the skin and mucous membranes affect the healing ability of the ulcer; the wounds are not healed for a long time, or recurrent after pseudo-healing, which seriously affects the health of the patient.

In recent years, with the vigorous development of biology, people have become more and more clear about the pathogenesis of tuberculosis, but the recent research is more concentrated on tuberculosis research [1]. At present, research on cutaneous tuberculosis is rare. Although there have been many 
reports highlighting risk factors and cytokine expression in various forms of EPTB, extrapulmonary tuberculosis[2, 3], knowledge of changes in pathogen-associated tissue expression in hosts is very inadequate.So far, only a few reports have studied chemokines and chemokine receptors in human tissues, which are responsive in TB patients[4].

Understanding an important aspect of host-pathogen interaction is to identify host tissue responses to infection and to identify causal factors associated with disease performance.

Chemokines are superfamilies of small molecular weight proteins (8 to $10-\mathrm{kd}$ ) with $20 \%$ to $70 \%$ homology in the amino acid sequence, which are involved in cell activation and proliferation and leukocyte recruitment to sites of inflammation such as ligands. They make an important impact. The receptor (CXCL10 / CXCR3) is involved in the selective migration of lymphocytes to the intestinal mucosa[5]. They are subdivided into families based on the relative positions of cysteine residues. There are at least four chemokine families, but only two are widely characterized.

\section{Methods}

\section{Tissue samples}

In this study, we included 14 patients (E1-E14) who were diagnosed with cutaneous tuberculosis at the Integrated Chinese and Western Medicine Hospital in Nanjing, Jiangsu Province, China. None of these patients had anti-tuberculosis treatment and no active tuberculosis, mental illness, impaired immune function (symptomatic HIV infection, confirmed or suspected HIV infection, leukemia, lymphoma or systemic malignancy), immunosuppressive therapy (corticosteroids, alkylating agents, antimetabolites, radiation therapy). In addition, 14 non-specific infectious ulcer patients (E15-E24) matched with cutaneous tuberculosis patients, such as age and gender, were included for comparative study.(Table 1) Among them, 6 patients with E1-E3 and E8-E10 were selected for local lesion tissue transcription analysis of CXCR3, CXCL9, CXCL0, CXCL11 mRNA expression. Finally, the differential expression levels of CXCR3, CXCL9, CXCL0 and CXCL11 chemokines in the local lesions of these 28 patients were verified by quantitative PCR. Table 1 summarizes the demographic and clinical characteristics of all patients with cutaneous tuberculosis and non-specific infectious ulcers. Informed consent was obtained from all patients or legal guardians. This study was approved by the hospital ethics committee.

Table 1 Details of cutaneous tuberculosis patients and Non-specific infectious ulcer patients enrolled in the study 


\begin{tabular}{|c|c|c|c|c|c|c|c|}
\hline Patient ID & Age & Sex & H/E Staining & Culture & $\begin{array}{l}\text { Current } \\
\text { Medication }\end{array}$ & $\begin{array}{c}\text { History } \\
\text { of TB }\end{array}$ & Microarray \\
\hline E-1 & 22 & $\mathrm{~F}$ & ¿ve & \ve & NO & NO & YES \\
\hline E-2 & 43 & $\mathrm{~F}$ & घve & पve & $\mathrm{NO}$ & NO & YES \\
\hline E-3 & 27 & $\mathrm{~F}$ & घve & पve & NO & NO & YES \\
\hline E-4 & 36 & $\mathrm{~F}$ & पve & $\square v e$ & $\mathrm{NO}$ & NO & $\mathrm{NO}$ \\
\hline E-5 & 26 & $\mathrm{~F}$ & घve & पve & $\mathrm{NO}$ & $\mathrm{NO}$ & $\mathrm{NO}$ \\
\hline E-6 & 26 & $\mathrm{M}$ & घve & पve & $\mathrm{NO}$ & $\mathrm{NO}$ & $\mathrm{NO}$ \\
\hline E-7 & 44 & $\mathrm{M}$ & घve & $\square \mathrm{ve}$ & NO & $\mathrm{NO}$ & NO \\
\hline E-8 & 31 & $\mathrm{~F}$ & घve & पve & NO & NO & $\mathrm{NO}$ \\
\hline E-9 & 22 & $\mathrm{M}$ & ¿ve & पve & $\mathrm{NO}$ & NO & NO \\
\hline E-10 & 59 & $\mathrm{~F}$ & घve & पve & $\mathrm{NO}$ & $\mathrm{NO}$ & $\mathrm{NO}$ \\
\hline E-11 & 44 & $\mathrm{M}$ & घve & पve & NO & NO & $\mathrm{NO}$ \\
\hline E-12 & 37 & $\mathrm{~F}$ & घve & पve & $\mathrm{NO}$ & $\mathrm{NO}$ & $\mathrm{NO}$ \\
\hline E-13 & 58 & $\mathrm{~F}$ & घve & ఫve & $\mathrm{NO}$ & $\mathrm{NO}$ & $\mathrm{NO}$ \\
\hline E-14 & 38 & $\mathrm{M}$ & घve & पve & NO & NO & NO \\
\hline E-15 & 33 & $\mathrm{~F}$ & पve & पve & $\mathrm{NO}$ & NO & YES \\
\hline E-16 & 24 & $\mathrm{~F}$ & घve & पve & $\mathrm{NO}$ & NO & YES \\
\hline E-17 & 60 & $\mathrm{M}$ & घve & पve & $\mathrm{NO}$ & $\mathrm{NO}$ & YES \\
\hline E-18 & 46 & $\mathrm{M}$ & घve & पve & $\mathrm{NO}$ & $\mathrm{NO}$ & $\mathrm{NO}$ \\
\hline E-19 & 39 & $\mathrm{~F}$ & $\square \mathrm{ve}$ & पve & $\mathrm{NO}$ & NO & $\mathrm{NO}$ \\
\hline E-20 & 63 & $\mathrm{~F}$ & पve & पve & $\mathrm{NO}$ & $\mathrm{NO}$ & $\mathrm{NO}$ \\
\hline E-21 & 34 & $\mathrm{M}$ & पve & पve & $\mathrm{NO}$ & NO & $\mathrm{NO}$ \\
\hline E-22 & 23 & $\mathrm{~F}$ & घve & पve & NO & NO & $\mathrm{NO}$ \\
\hline E-23 & 42 & $\mathrm{~F}$ & पve & पve & $\mathrm{NO}$ & NO & $\mathrm{NO}$ \\
\hline E-24 & 28 & $\mathrm{~F}$ & घve & ¿ve & NO & NO & NO \\
\hline E-25 & 33 & $\mathrm{~F}$ & घve & पve & NO & NO & $\mathrm{NO}$ \\
\hline E-26 & 32 & $\mathrm{~F}$ & ¿ve & Øve & NO & NO & NO \\
\hline
\end{tabular}




\begin{tabular}{|c|c|c|c|c|c|c|c|}
\hline E-27 & 21 & M & $\square v e$ & ave & NO & NO & NO \\
\hline E-28 & 47 & M & ave & वve & NO & NO & NO \\
\hline
\end{tabular}

\section{RNA extraction}

For RNA purification, we used TRIzol reagent (Invitrogen, Grand Island, NY, USA) according to the manufacturer's instructions, and then applied PBMC to the RNeasy spin column (Qiagen, Venlo, Limburg, Netherlands). Quantitative and quality assessments were performed by using Nanodrop and an Agilent 2100 Bioanalyzer (Agilent Technologies, Santa Clara, CA, USA), respectively.

\section{IncRNA microarray}

Global spectra of human mRNA, circRNA, IncRNA and protein-encoding transcripts were designed using Human IncRNA Microarray V6.0 (Agilent Technologies, Santa Clara, CA, USA). A third generation IncRNA microarray detected approximately 30,586 IncRNAs and 26,109 encoded transcripts. A public transcriptome database (RefSeq, UCSC known genes and gene coding, etc.) and IncRNA were used. Specific exons or splicing junction probes are used to accurately identify each transcript of each transcript. In addition, positive probes for housekeeping genes and negative probes were printed onto the array for hybridization quality control. GeneSpring GX v11.5.1 software (Agilent Technologies) is used to extract and standardize data. Volcano Plot filtration and hierarchical clustering were used to identify differentially expressed IncRNA and mRNA, which reached statistically significant levels. Differentially expressed IncRNA and mRNA were identified by a random variance model, where the $P$ values were calculated by paired t-test. The significant cutoff values for up-and down-regulated IncRNA and mRNA were fold change $\geq 2.0, P \leq 0.05$.

\section{Quantitative Real-time PCR}

To further verify the credibility of gene sequencing results, CXCR3, CXCL9, CXCL0, and CXCL11 were selected for PCR validation. We selected 14 patient samples (E1-E14) and 14 control samples (E15-E24) (added 4 patient samples not used for microarray experiments and 4 normal human samples not used for microarray experiments).

$50 \mathrm{ng}$ of RNA was reverse transcribed into cDNA using Vazyme HiScript II QRT SuperMix for qPCR following manufacturer's recommendations. RT-PCR was performed with StepOnePlus Real-time PCR System (ABI) 96 well plate (LIFE) according to manufacturer's protocols. The GAPDH rRNA genes were used for normalization and fold changes were calculated using the formula, 2-( $\Delta \Delta \mathrm{Ct})$. 


\section{Results}

\section{Patient characterization}

Patients with clinical cutaneous tuberculosis underwent a prospective study at the Integrated Chinese and Western Medicine Hospital in Nanjing, Jiangsu Province, China. The clinical manifestations were pale edema of the granuloma of the wound, purulent necrosis on the surface, unclear edge of the ulcer, dark red skin, white flocculent necrotic tissue like bean dregs in the abscess. Or the sinus opening can be seen and there are multiple branches extending in different directions. The wound is difficult to heal. During the course of the disease, the patient developed fatigue, fever, dizziness, night sweating, anorexia and weight loss.[4]

In this study, a total of 28 patients (E1-E28) who met the inclusion criteria were enrolled in the study. Ulcer tissue was obtained from all patients and HE staining (pathological diagnosis) was performed. Among them, 14 patients (E1-E14) were positive for HE staining and histopathologically positive (Fig. 1). They were treated with anti-tuberculosis drugs. Another 14 (E15-E28) control patients were non-specific infectious ulcers.

\section{Expression profile of human local inflammatory granulation tissue}

We performed a transcriptomic analysis of three experimental groups (E1-E3) and three control patients (E15-E17) using a whole human genomic oligonucleotide array. Based on this analysis, we identified 10,261 probes showing differential expression (adjusted p-value $<0.05$, fold-value $\pm 1.2(\approx \log F \mathrm{C} \pm 0.27)$, of which 2,594 genes had lower mRNA expression than the control group, 1,802 The gene expression was higher than that of the control group (Table 2). The relationship between gene expression profiles, the principal component analysis of the normalized gene expression data of the sample and the use of all unsupervised hierarchical clustering to examine the relationship between gene expression profiles. The largest variation in the data set (Fig. 2).

Table 2 Differential screening gene statistics in the study

\begin{tabular}{|c|c|c|c|c|c|c|c|c|c|c|c|c|c|}
\hline & & \multicolumn{3}{|c|}{ mRNA $\square$ Regulated $\square$} & \multicolumn{3}{|c|}{ lncRNA』Regulated $\square$} & \multicolumn{3}{|c|}{ Multiple_Complex $\square$ Regulated $\square$} & \multicolumn{3}{|c|}{ circRNA $\square$ Regulated $\square$} \\
\hline & & Up & Down & Total & Up & Down & Total & Up & Down & Total & Up & Down & Total \\
\hline Case & Control & 1802 & 2594 & 4396 & 2314 & 1282 & 3596 & 690 & 1204 & 1894 & 302 & 73 & 375 \\
\hline
\end{tabular}

\section{Upregulation and verification of chemokines in skin ulcer tissue}

Chemokines are important components that direct immune cells to the site of infection and promote disease progression. Microarray results showed that mRNA expression in skin ulcer tissue was different 
from that in non-specific infectious ulcer local tissues, and tuberculosis-related genes were confirmed. This method provides expression data for 4,396 mRNAs showing up to 1802 genes upregulated in local inflammatory granulation tissue in cutaneous tuberculosis. The mRNA differential screening results showed that CXCL9, CXCL10, CXCL11 and CXCR3 were the most prominent upregulated chemokines. CXCL10 chemotactic leukocytes accumulate in the inflammation site, and CXCL10 binds to CXCR3 and exerts chemotaxis on T cells.(Fig.3)

\section{GO and KEGG analyses}

GO and KEGG analyses were performed to obtain detailed information on the biological functions and potential mechanisms of these differentially expressed mRNAs. A total of 4396 filtered mRNAs (2-fold change) were included in GO and KEGG analyses. GO analysis revealed that 1802 upregulated mRNAs in biological processes were involved in cellular responses to immune responses, adaptive immune response, interferon-gamma-mediated signaling pathway, inflammatory response, innate immune response, $T$ cell costimulation regulation of immune response T cell activation and so on (Fig. 4) and in molecular function were analyzed tumor necrosis factor receptor binding, receptor activity, chemokine receptor activity (Fig. 5) and In cellular component were nucleosome, external side of plasma membrane, nuclear (Fig. 6). However, 2594 downregulated mRNAs were participants mitochondrial respiratory chain complex I assembly, muscle contraction, G0:0006120, tricarboxylic acid cycle (Fig. 7). KEGG analysis of the 1802 upregulated mRNAs Natural killer cell mediated cytotoxicity, Graft-versus-host disease, Chemokine signaling pathway, Antigen processing and presentation (Fig. 8).

\section{Real-time quantitative RT-PCR verification sequencing results}

The expression differences of CXCR3, CXCL9, CXCL10 and CXCL11 genes were detected by qRT-PCR. Compared with the control group, both CXCL9,CXCL10,CXCL11,CXCR3 were significantly upregulated(Fig.9).

\section{Discussion}

Tuberculosis is one of the top ten causes of infectious diseases that endanger human health and cause human death. People with AIDS often die from tuberculosis infection. China is a country with a high burden of tuberculosis.

Lymphatic tuberculosis (LNTB) is the most common form of extrapulmonary tuberculosis, leading to lymphadenopathy cutaneous tuberculosis. Cutaneous tuberculosis is often referred to as tuberculous ulcer in China.

However, the lack of a clear pathogenesis hinders diagnosis and treatment. Reported CXCL9, CXCL10 mRNA expression levels associate with cutaneous tuberculosis. Literature reported, IFN-g signaling, via Tbet, also promotes CXCR3 expression on a subset of Th1-specific regulatory T cells[6]. The chemokine receptor CXCR3 is a $\mathrm{G}$ protein-coupled receptor found predominantly on $\mathrm{T}$ cells that is activated by three 
ligands as follows: CXCL9 (Mig), CXCL10 (IP-10), and CXCL11 (I-TAC). Three ligands, CXCL11 is the most potent inducer of CXCR3 internalization and is the physiologic inducer of CXCR3 internalization after T cell contact with activated endothelial cells[7]. C-X-C motif chemokine 10 (CXCL10) is also known as IP10. The biological function of CXCL10 is to induce chemotaxis, cell growth, angiogenesis, and apoptosis by binding to its surface chemokine receptor CXCR3, and CXCL10 is recognized as an inflammatory chemokine. Moreover, CXCL10 plays an important role in various pathological states. CXCL10 had been reported to act as a marker for the diagnosis of tuberculosis $[8,9]$.The production of CXCL9 is specifically induced by IFN- $\mathrm{Y}[10]$,and IFN- $\mathrm{y}$ plays an important role in the pathogenesis of tuberculosis-related diseases.[11]

Specific blockage of CXCR3 (a CXCL10 receptor) significantly decreased the severity of chronic pulmonary inflammation by decreasing the recruitment of inflammatory cells. [12] Similarly, mRNA CXCL10 expression was up-regulated in tuberculous ulcer tissues, namely Log FC.

Given their pleiotropic nature, CXCR3 ligands play their part in a myriad of diseases. Involvement of all CXCR3 ligands has been observed in various angiogenesis-related pathologies as well as immunological disorders, the latter being mostly related to autoimmunity or infection.

In this experiment, a microarray of whole genome mRNA expression profiles of local inflammatory granulation tissue of cutaneous tuberculosis patients was compared with local inflammatory granulation tissue of non-specific infectious ulcer patients.

However, our detection found that the mRNA expression levels of CXCR3, CXCL9, CXCL10, CXCL11 were upregulated in cutaneous tuberculosis. And CXCR3, CXCL9, CXCL10, CXCL10 verificatied by QPCR. It was found that CXCR3, CXCL9, CXCL10, and CXCL10 were up-regulated in inflammatory granulation tissue of cutaneous tuberculosis, indicating that up-regulation of CXCR3, CXCL9, CXCL10, and CXCL10 can be used as a diagnostic marker for tuberculous ulcer. GO analysis and KEGG analysis also indicated that activation of various immune responses and inflammatory responses may be associated with upregulation of chemokine genes. The high expression of chemokine CXCL10 in inflammatory granulation tissue of cutaneous tuberculosis is positively correlated with immune response. In addition, further research is needed to assess their monitoring of the efficacy of cutaneous tuberculosis treatment. Our goal is to investigate the potential utility of mRNA as a biomarker or therapeutic target for cutaneous tuberculosis.

\section{Conclusion}

This study aims to summarize what we know about chemokines involved in the immune response and inflammation of cutaneous tuberculosis in human diseases, all of which are essential for advancing hostpathogen interactions that affect disease outcomes.

\section{Abbreviations}


Mtb: M. tuberculosis; GO: gene ontology; KEGG: Kyoto Gene and Genomic Encyclopedia; IncRNA: Long non-coding RNA;TB :tuberculosis ;BCG: bacillus Calmette-Guérin; qRT-PCR: Quantitative Real-time PCR; LNTB: Lymphatic tuberculosis;

\section{Declarations}

\section{Acknowledgments}

Not applicable.

\section{Authors' contributions}

ZH was responsible for the conception and design of the study. JQ,LG,YY,SZ and MG were responsible for the acquisition and analysis of data; furthermore, JQ and LG were in charge of statistical analysis. ZiHui Huang drafted the manuscript and $\mathrm{LH}, \mathrm{YL}$ revised it. All of the authors listed on the by-line have read and approved the manuscript.

\section{Funding}

This work was supported by the Nanjing key medical science and technology development project (ZKX16067); Nanjing health young talents program (QRX17030); Science and technology project of jiangsu Chinese medicine and pharmacy bureau (YB2017042) in study design, data collection and analysis, decision to publish, or preparation of the manuscript.

\section{Availability of data and materials}

Not applicable.

\section{Ethics approval and consent to participate}

The present study was approved by the ethics committee of Nanjing integrated traditional Chinese and western medicine hospital『NO:201901001》. All subjects provided informed consent for this study, which was approved by the Ethics Committee of Nanjing integrated traditional Chinese and western medicine hospital. This project was performed in accordance with the approved guidelines from the ethical principles outlined in the Declaration of Helsinki.

\section{Consent for publication}

Not applicable.

\section{Competing interests}

The authors declare that they have no competing interests.

\section{Author details}


${ }^{1}$ Nanjing Hospital of Integrated Traditional Chinese and Western Medicine affiliated to Nanjing University of Traditional Chinese Medicine, No.179 Xiaolingwei, Xuanwu District, Nanjing 210014, Jiangsu, China

${ }^{2}$ Nanjing University of Traditional Chinese Medicine, No.138 Xianlin Avenue, Qixia District, Nanjing 210043, Jiangsu, China

\section{References}

1. Anil S, Ellepola AN, Samaranayake LP, Beena VT: Tuberculous ulcer of the tongue as presenting feature of pulmonary tuberculosis and HIV infection. Gen Dent 2000;48(4):458-461.

2. Hasan Z, Jamil B, Khan J, Ali R, Khan MA, Nasir N, Yusuf MS, Jamil S, Irfan M, Hussain R: Relationship between circulating levels of IFN-gamma, IL-10, CXCL9 and CCL2 in pulmonary and extrapulmonary tuberculosis is dependent on disease severity. SCAND J IMMUNOL 2009;69(3):259267.https://doi.org/10.1111/j.1365-3083.2008.02217.x.

3. Pavan KN, Anuradha R, Andrade BB, Suresh N, Ganesh R, Shankar J, Kumaraswami V, Nutman TB, Babu S: Circulating biomarkers of pulmonary and extrapulmonary tuberculosis in children. CLIN VACCINE IMMUNOL 2013;20(5):704-711.https://doi.org/10.1128/CVI.00038-13.

4. Maji A, Misra R, Kumar MA, Kumar D, Bajaj D, Singhal A, Arora G, Bhaduri A, Sajid A, Bhatia S et al: Expression profiling of lymph nodes in tuberculosis patients reveal inflammatory milieu at site of infection. Sci Rep 2015;5:15214. https://doi.org/10.1038/srep15214.

5. Hardaker EL, Bacon AM, Carlson K, Roshak AK, Foley JJ, Schmidt DB, Buckley PT, Comegys M, Panettieri RJ, Sarau HM et al: Regulation of TNF-alpha- and IFN-gamma-induced CXCL10 expression: participation of the airway smooth muscle in the pulmonary inflammatory response in chronic obstructive pulmonary disease. FASEB J 2004;18(1):191-193.https://doi.org/10.1096/fj.03-0170fje.

6. Kanda N, Shimizu T, Tada Y, Watanabe S: IL-18 enhances IFN-gamma-induced production of CXCL9, CXCL10, and CXCL11 in human keratinocytes. EUR J IMMUNOL 2007;37(2):338-350. https://doi.org/10.1002/eji.200636420.

7. Xanthou G, Duchesnes CE, Williams TJ, Pease JE: CCR3 functional responses are regulated by both CXCR3 and its ligands CXCL9, CXCL10 and CXCL11. EUR J IMMUNOL 2003;33(8):2241-2250. https://doi.org/10.1002/eji.200323787.

8. Marino S, Myers A, Flynn JL, Kirschner DE: TNF and IL-10 are major factors in modulation of the phagocytic cell environment in lung and lymph node in tuberculosis: a next-generation twocompartmental model. J THEOR BIOL 2010;265(4):586598.https://doi.org/10.1016/j.jtbi.2010.05.012.

9. Strzelak A, Komorowska-Piotrowska A, Ziolkowski J: [CXCL10/IP-10 as a new biomarker for Mycobacterium tuberculosis infection]. Pol Merkur Lekarski 2012;33(198):342-345.

10. Mizuta M, Shimizu M, Inoue N, Nakagishi Y, Yachie A: Clinical significance of serum CXCL9 levels as a biomarker for systemic juvenile idiopathic arthritis associated macrophage activation syndrome. CYTOKINE 2019;119:182-187. https://doi.org/10.1016/j.cyto.2019.03.018. 
11. Lv Q, He C, Quan F, Yu S, Chen X: Corrigendum to "DOX/IL-2/IFN-gamma co-loaded thermo-sensitive polypeptide hydrogel for efficient melanoma treatment". Bioact Mater 2019;4:167.

https://doi.org/10.1016/j.bioactmat.2019.04.002

12. Gao B, Lin J, Jiang Z, Yang Z, Yu H, Ding L, Yu M, Cui Q, Dunavin N, Zhang M et al: Upregulation of chemokine CXCL10 enhances chronic pulmonary inflammation in tree shrew collagen-induced arthritis. Sci Rep 2018;8(1):9993. https://doi.org/10.1038/s41598-018-28404-y.

\section{Figures}
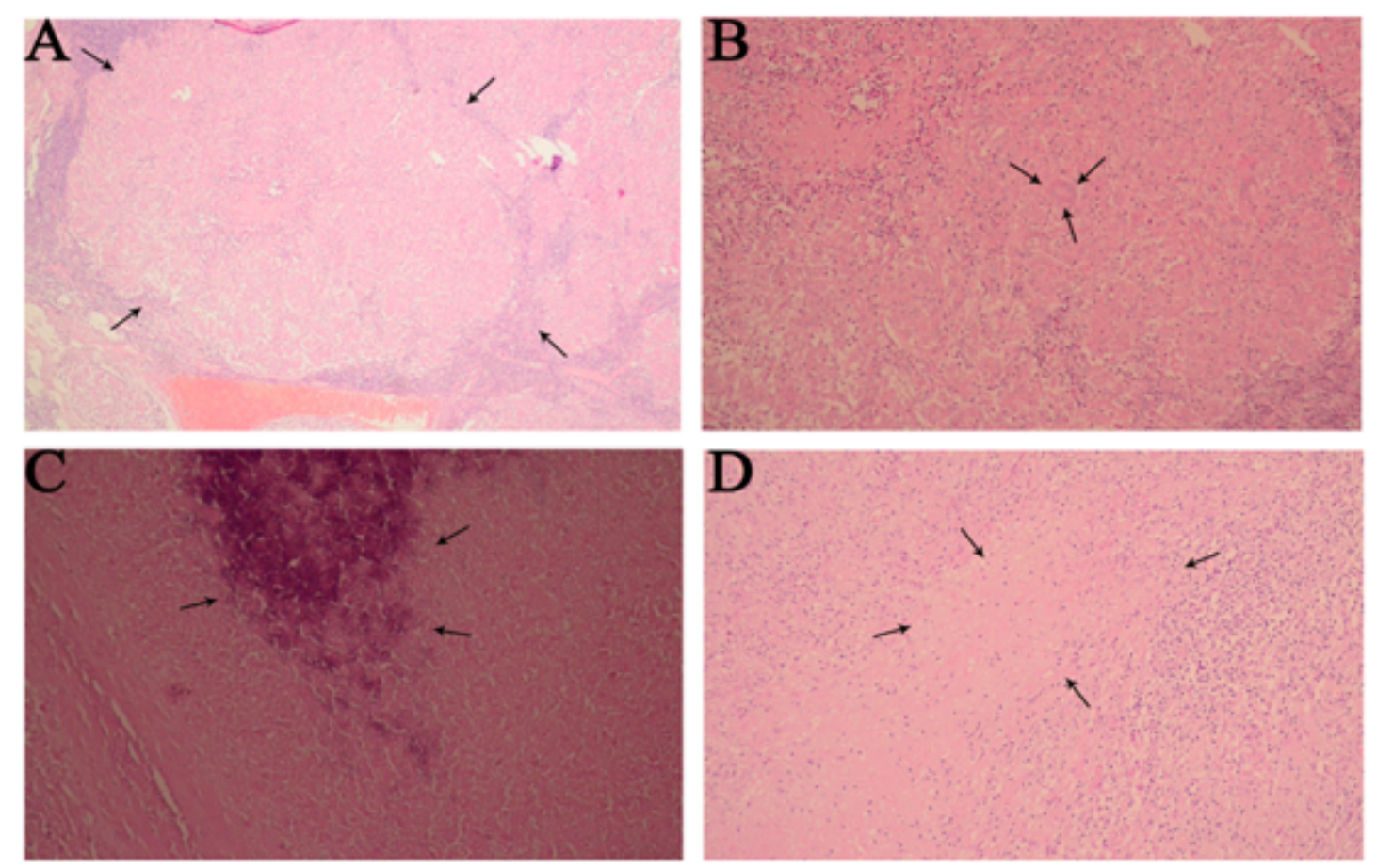

Figure 1

Hematoxylin and eosin staining for identification of granuloma structures. Hematoxylin and eosin stained sections of lymph nodes in patients infected with $M$. tuberculosis (A) large area coagulative necrosis with massive neutrophil infiltration (200X) (B) granulomatosis (arrow mark) and Langhans giant cells (The arrow is highlighted). Large area of coagulative necrosis with massive neutrophil infiltration (400X) (C) large area coagulative necrosis with focal calcification (400X) (D) This is an epithelioid granuloma with coagulative necrosis in the center, surrounding Epithelioid cells have scattered lymphocytes and neutrophils. (200X) 


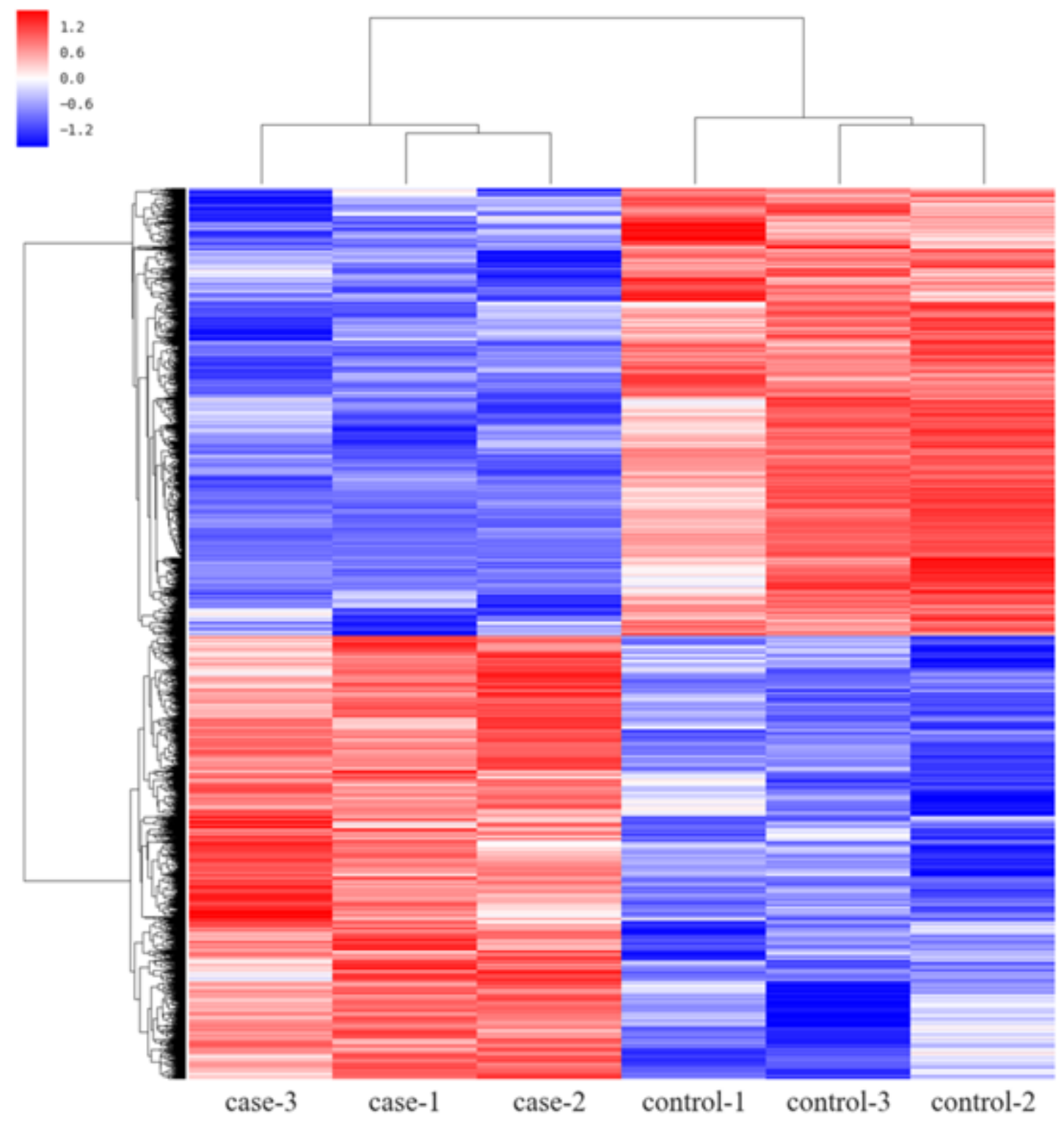

\section{Figure 2}

Cluster analysis of differential expression of transcriptome analysis of cutaneous tuberculosis inflammatory granulation tissue mRNA of cutaneous tuberculosis infected tissue patients and nonspecific infectious ulcer control 


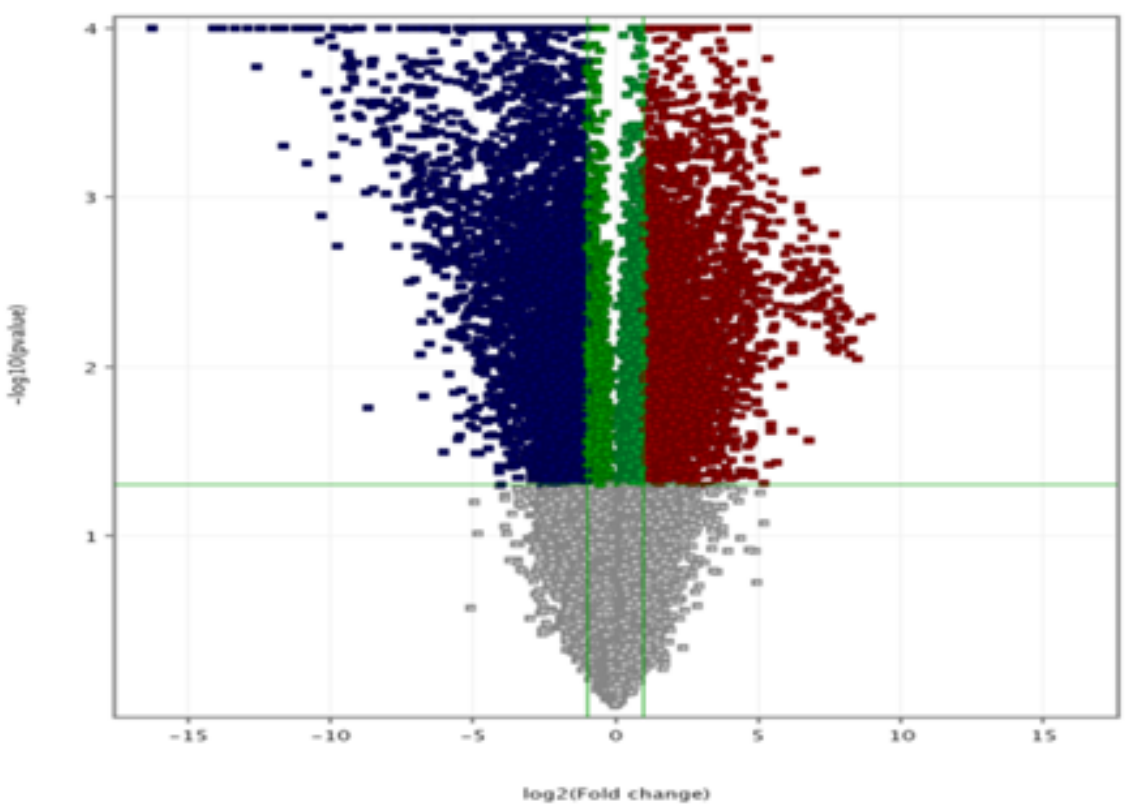

Figure 3

Transcriptome analysis of cutaneous tuberculosis infected tissues. The volcano map shows differentially expressed genes (DEG) in the microarray analysis of cutaneous tuberculosis infected tissues relative to control tissues. The p-value of the false discovery rate (FDR) adjustment of the negative log 10 transformation is plotted against the average log2 fold change of the expression ( $x$-axis). The DEG was measured with limma and then corrected with FDR. The horizontal dashed line indicates the significance threshold (FDR adjustment $P<0.05$ ), and the vertical dashed line indicates the up (right) and down (left) probes. 


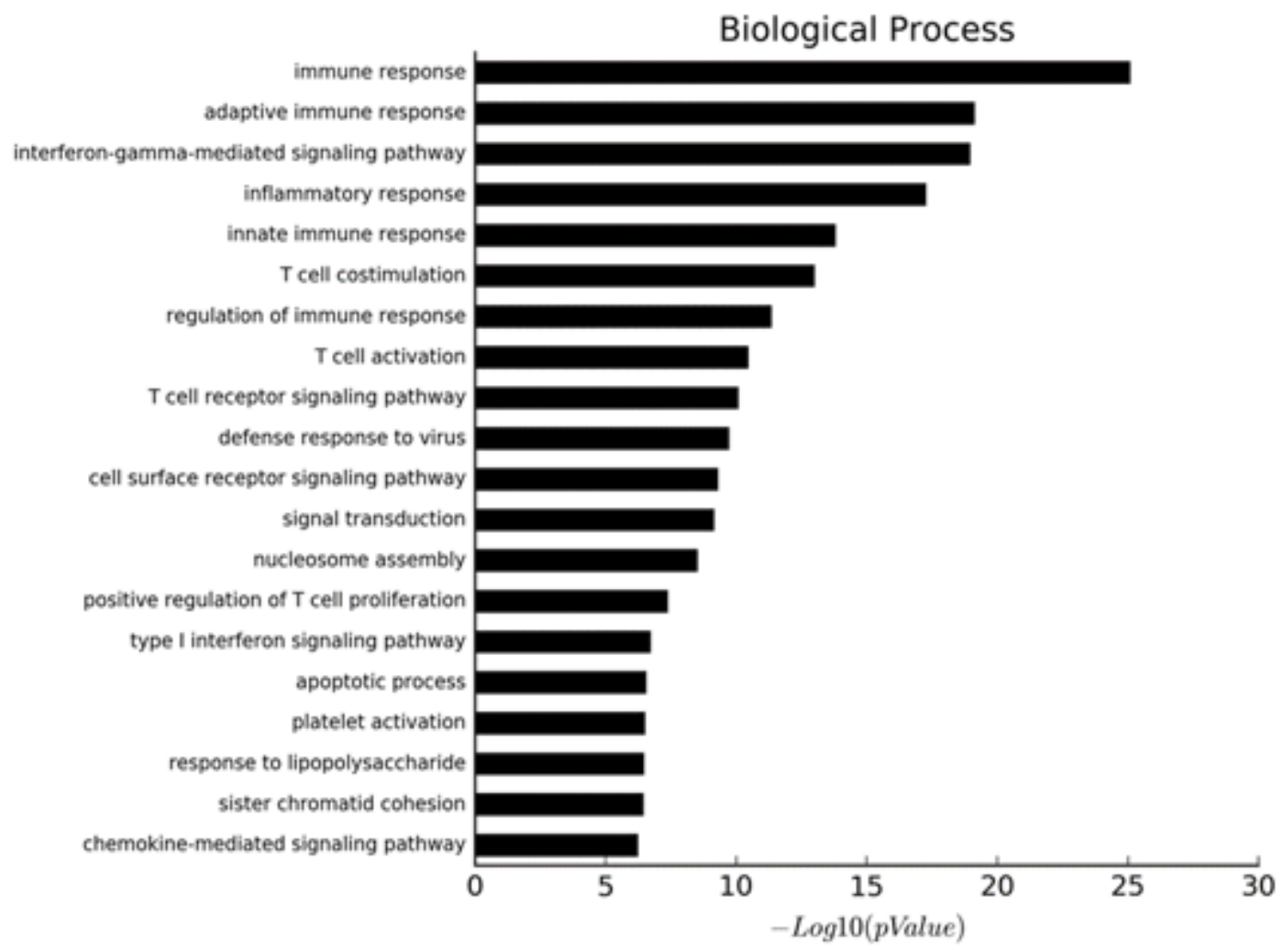

Figure 4

Transcriptome analysis of cutaneous tuberculosis inflammatory granulation tissue. The first $20 \%$ of David and the biological process analysis of upregulation of DEG. The bar graph shows the number of DEGs[4] observed in each gene ontology (at least 20 genes per class), and the corresponding $p$-values are represented in the first axis.

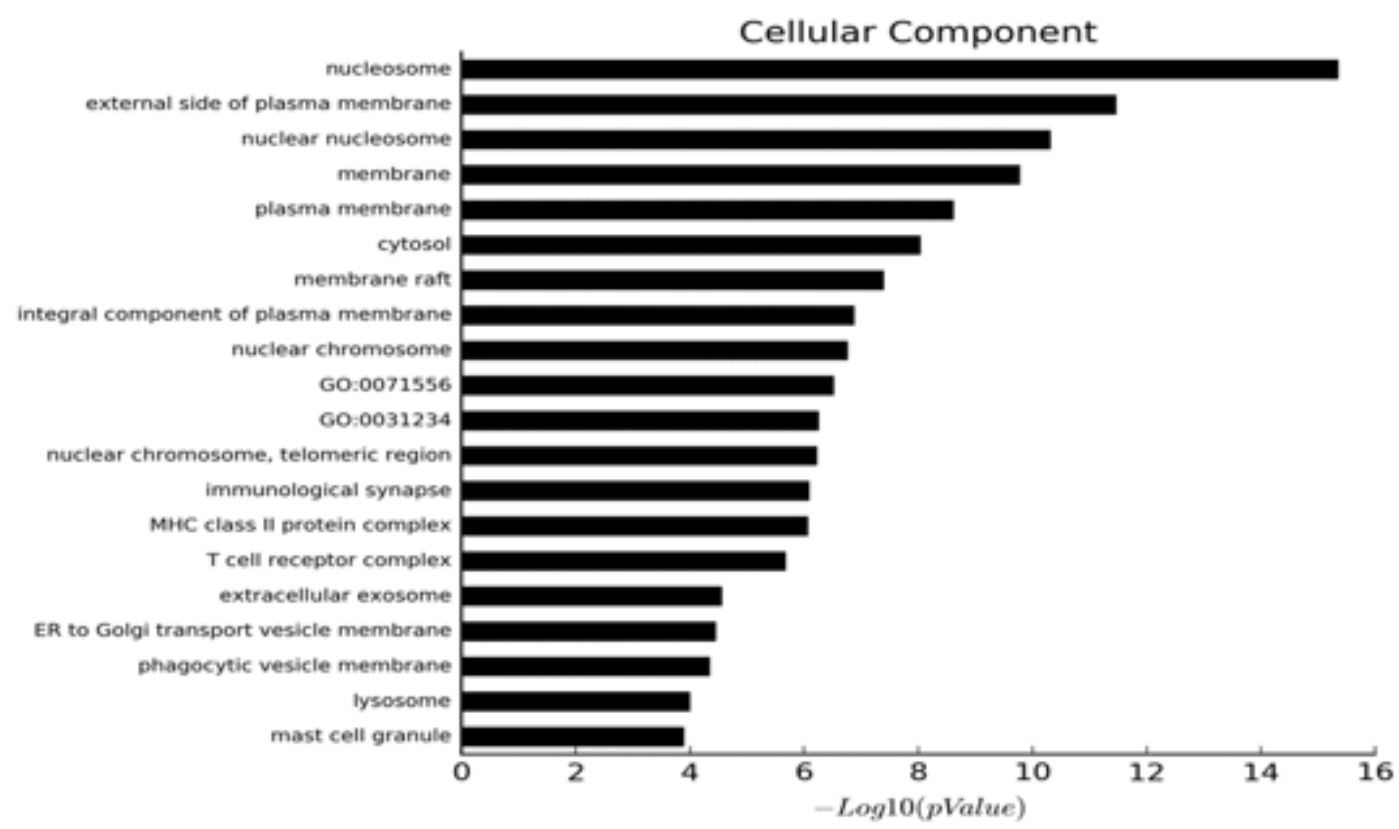




\section{Figure 5}

Transcriptome analysis of cutaneous tuberculosis inflammatory granulation tissue Analysis of the first $20 \%$ of the large intestine and the Celluar component of up-regulated DEG. The bar graph shows the number of DEGs [4] observed in each gene ontology (at least 20 genes per class), and the corresponding p-values are represented in the first axis.

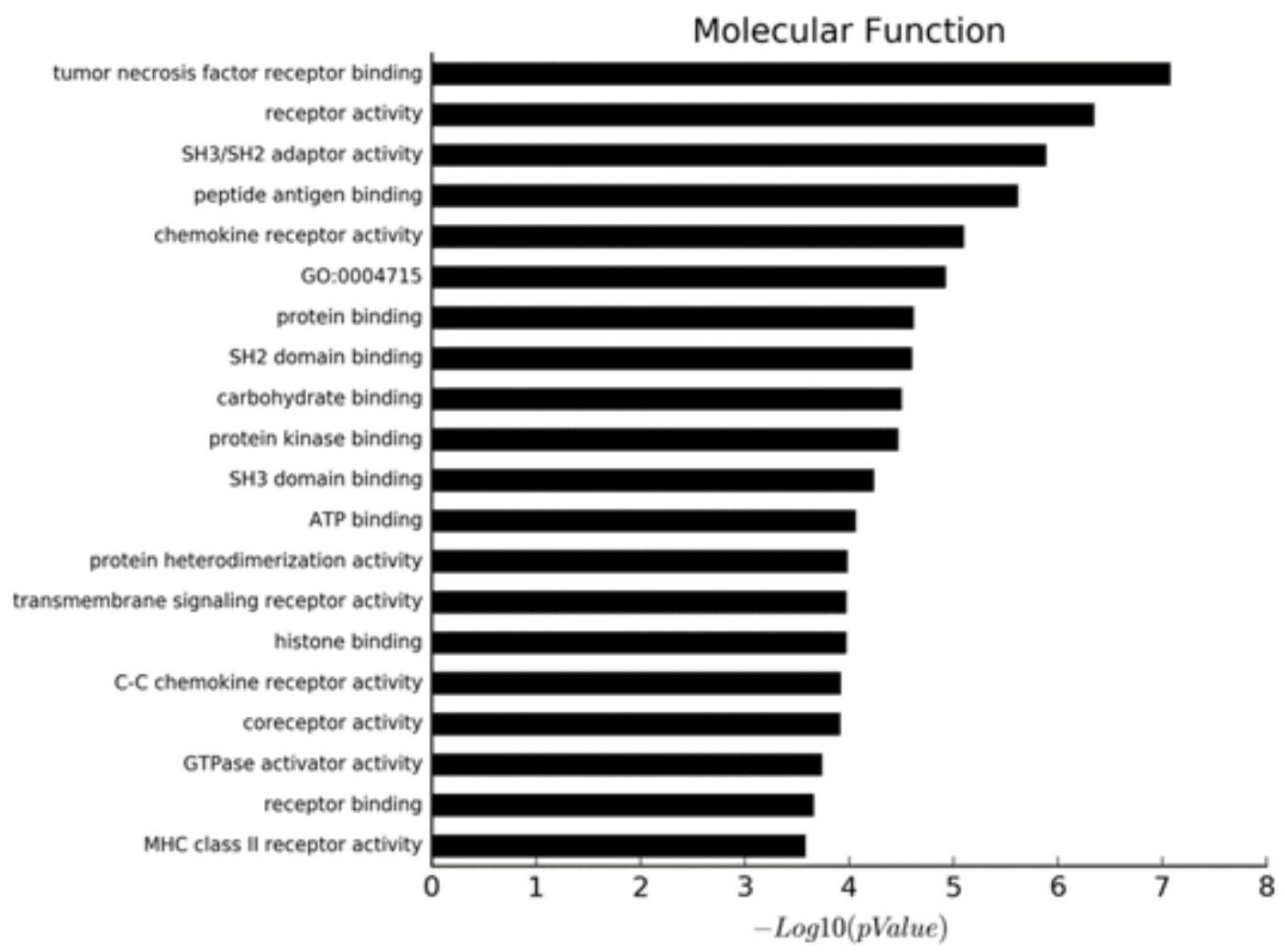

\section{Figure 6}

Transcriptome analysis of cutaneous tuberculosis inflammatory granulation tissue. Molecular functional analysis of the top $20 \%$ of the large intestine and up-regulation of DEG. The bar graph shows the number of DEGs[4]observed in each gene ontology (at least 20 genes per class), and the corresponding $p$-values are represented in the first axis. 


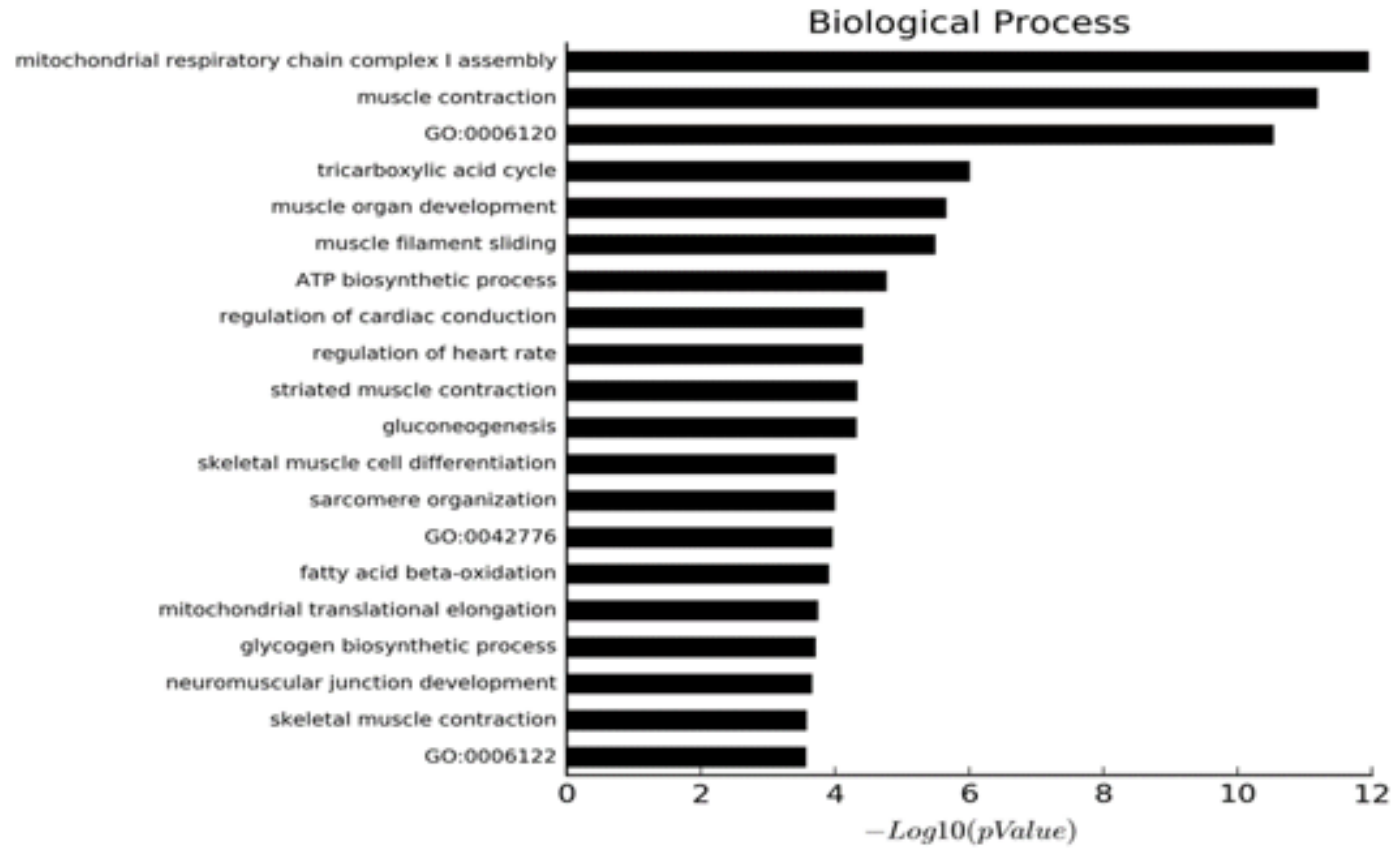

\section{Figure 7}

Transcriptomic analysis of cutaneous tuberculosis inflammatory granulation tissue. The first $20 \%$ of David's biological process analysis of down-regulation of DEGs. The bar graph shows the number of DEGs[4] observed in each gene ontology (at least 20 genes per class), and the corresponding p-values are represented in the first axis.

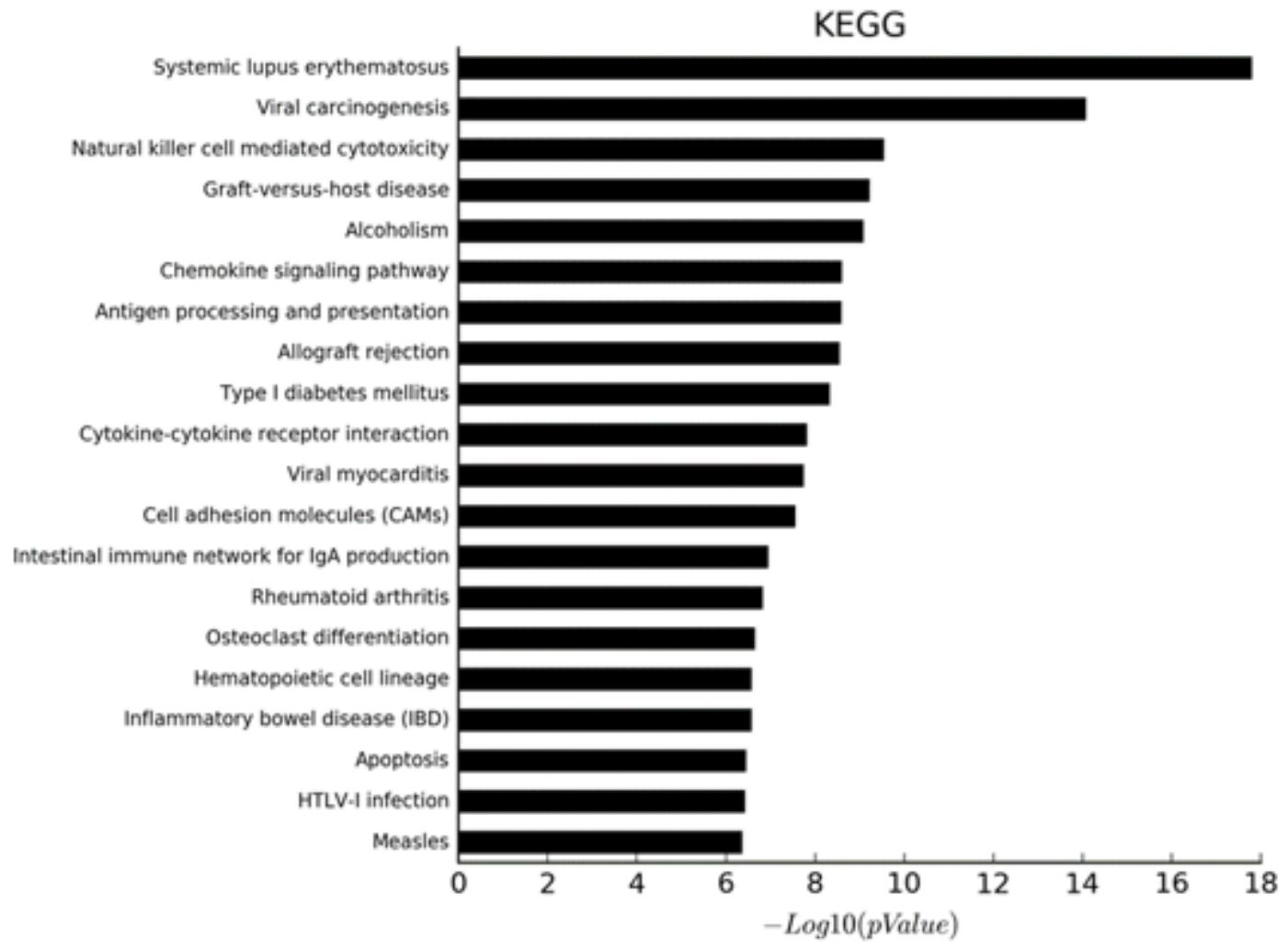


Figure 8

Twenty pathways of transcriptomic analysis of cutaneous tuberculosis inflammatory granulation tissue patients compared to non-specific infectious ulcer patient controls.

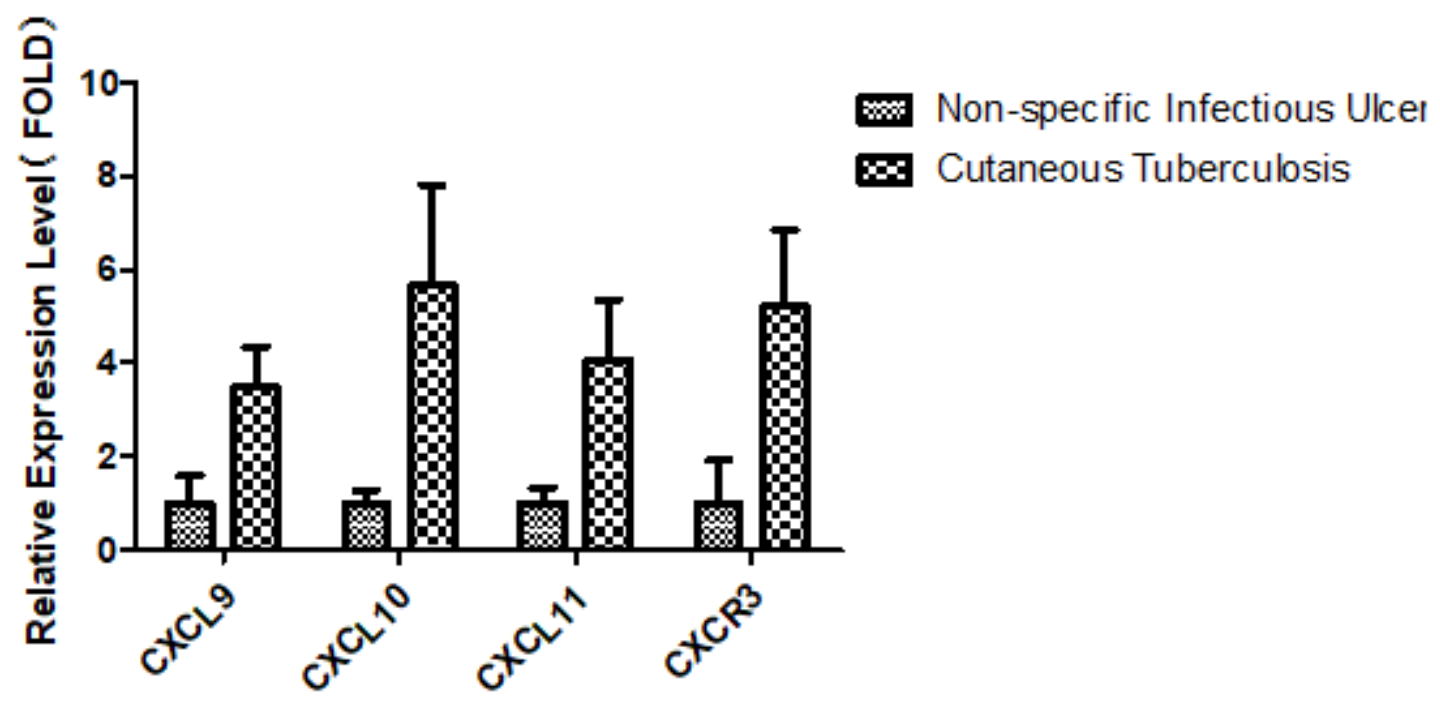

\section{Figure 9}

Real-time quantitative RT-PCR verification sequencing results

\section{Supplementary Files}

This is a list of supplementary files associated with this preprint. Click to download.

- Rawdata.pdf

- Rawdata1.pdf 